Fatemeh Abbasi, J. Jay Leitch, Zhangfei Su, Grzegorz Szymanski and Jacek Lipkowski “Direct visualization of alamethicin ion pores formed in a floating phospholipid membrane supported on a gold electrode surface" Electrochim Acta, 2018,267,195-205.

\title{
Direct visualization of alamethicin ion pores formed in a floating phospholipid membrane supported on a gold electrode surface
}

\author{
Fatemeh Abbasi, J. Jay Leitch, Zhangfei Su, Grzegorz Szymanski and Jacek Lipkowski* \\ Department of Chemistry, University of Guelph, Guelph, Ontario, N1G 2W1, Canada
}

\begin{abstract}
Unilamellar DMPC/DMPG vesicles in the absence and presence of alamethicin were fused onto the surface of a gold electrode modified with a 1-thio- $\beta$-D-glucose self-assembled monolayer. The resulting floating bilayer lipid membranes (fBLMs) were investigated using atomic force microscopy (AFM) and electrochemical impedance spectroscopy (EIS). A corrugated film structure was observed for the pure DMPC/DMPG fBLMs due to surface stress between the tightly packed lipids. These corrugations are removed by the addition of alamethicin suggesting the lipid-peptide interactions alleviate the overall surface stress creating a more uniform bilayer. Both DMPC/DMPG films in the absence and presence of alamethicin had thickness of $5.5 \pm 0.9$ $\mathrm{nm}$ demonstrating that alamethicin has a minimal effect on the overall bilayer thickness. However, a significant decrease in membrane resistivity was observed when alamethicin was inserted into the fBLM indicating that the peptides are forming ion conducting pores. A direct visualization of the alamethicin pores was obtained by molecular resolution AFM images revealing that the pores are not randomly dispersed throughout the bilayer, but instead form hexagonal aggregates. The diameter of an individual pore within the aggregates is equal to $2.3 \pm$ $0.3 \mathrm{~nm}$, which is consistent with the size of a hexameric pore predicted by molecular dynamics simulations. Additionally, the image revealed a broad size distribution of alamethicin aggregates,
\end{abstract}


which explains the origin of multiple conductivity states observed for the incorporation of alamethicin into free standing bilayer lipid membranes (BLMs).

\section{Introduction}

Alamethicin, a 20 amino acid residue antimicrobial peptide, was first discovered by Meyer and Reusser in the culture broth media of the fungus Trichoderma viride in 1976 [1]. Alamethicin belongs to the peptaibol family with an acetylated $\mathrm{N}$-terminus and a phenylalaninol (Pheol) residue at the $\mathrm{C}$-terminal end [2]. Peptaibol peptides are characterized by the presence of 2-aminoisobutyric acid (Aib), which induces a helical secondary structure. A proline residue at position 14 divides the peptide into a 13-residue $\mathrm{N}$-terminal $\alpha$-helix and a 7 -residue 310 -helix at C-terminus. The total length of the alamethicin peptide is $3.2 \mathrm{~nm}$, which is comparable to the thickness of the lipid of a biological cell membrane. The positions of the Aib and Gln residues on the alamethicin helices make the peptide amphipathic where one side is hydrophobic and the other side is more hydrophilic [3]. The difference in hydrophobicity provides a driving force for peptide adsorption at the biological membrane surface and insertion into the membrane core upon further aggregation with other alamethicin peptides[3].

The adsorption and insertion of the alamethicin depend on its peptide-to-lipid concentration. At low peptide concentrations, alamethicin adsorbs to the membrane surface with its helical axis oriented in the direction parallel to the surface ( $\mathrm{S}$ state). At sufficiently high peptide-to-lipid ratios ( $\mathrm{P} / \mathrm{L} \geq 1 / 15$ for most lipids), alamethicin inserts into bilayer (I state) [4,5]. In I state, the alamethicin molecules span the hydrophobic region of the bilayer and aggregate around a central pore with their hydrophobic domains in contact with the phospholipid tails. The polar regions of the peptides are oriented towards the center of the pore creating an aqueous channel that permits the transport of small ions across the bilayer [6]. This pore structure is referred to as the barrel-stave model and is responsible for the antimicrobial activity of alamethicin by allowing for the indiscriminate transport of ions across the bacterial cell membrane leading to cell lysis [6,7].

Electrochemical measurements (I-V curves) of the alamethicin-incorporated free standing bilayers (black lipid membranes) show multiple conductance steps providing evidence that the alamethicin pore consists of a varying number of peptide monomers [6]. A voltage-dependent 
conductance is observed in the alamethicin inserted black lipid membrane where the conductance is proportional to the ninth power of the bulk alamethicin concentration [8,9]. This voltagedependent conductance has been recently explained by Guidelli and Becucci [10] using model that assumes peptide insertion driven by the electric field-dipole interactions, followed by nucleation of peptide molecules into clusters forming transmembrane ion pores.

The structure of the alamethicin aggregates in model membranes has been extensively investigated by X-ray crystallography. Pan et al. [11] showed that the composition of phospholipid bilayers has an overall effect on the size of the aggregated alamethicin pore. In bilayers composed of 1,2-dioleoyl-sn-glycero-phosphatidylcholine (DOPC) and 10\% alamethicin, the pore consists of five alamethicin monomers with an outer radius of $1.37 \mathrm{~nm}$, while in membranes of 1,2-dierucoyl-sn-glycero-phosphatidylcholine (DEPC) with the same peptide concentration, the alamethicin pore is composed of $8-9$ monomers with an outer radius of $1.9 \mathrm{~nm}$. The length of the DEPC lipid chain is four carbons longer than the DOPC lipid implying that the aggregation is driven by the length of the lipid tails. These two lipids are unsaturated meaning that their corresponding bilayers are in the liquid crystalline state. He et al. [12] tested the effects of peptide aggregation in saturated phospholipid bilayers, specifically 1,2dilauroyl-sn-glycero-3-phosphatidylcholine (DLPC) and 1,2-diphytanoyl phosphatidylcholine (DPhPC). The alamethicin pore consisted of $8-9$ monomers with a radius of $2.0 \mathrm{~nm}$ in the DLPC gel state bilayer and 11 monomers with an outer pore radius of $2.5 \mathrm{~nm}$ in the DPhPC liquid crystalline bilayer. Salditt et al. [13] used X-ray scattering to measure the aggregation of alamethicin peptides in bilayers composed of both saturated and unsaturated phospholipid bilayers with variable acyl chain lengths. These studies show that alamethicin aggregation is largely dependent on the phase state and thickness of the phospholipid bilayer [13]. The Chen research group applied sum frequency generation (SFG) and the attenuated total reflection (ATR) spectroscopy to study alamethicin in POPC bilayers supported on a $\mathrm{CaF}_{2}$ prism $[14,15]$. They found that alamethicin was inserted into the lipid bilayer in the fluid phase, but remained on the external surface when the bilayer was in a gel state. Quartz crystal microbalance with dissipation monitoring (QCM-D) was used to study the interactions of alamethicin with the egg phosphatidylcholine (egg PC) supported lipid bilayer. QCM-D results support the model that the inserted peptides form a cylindrical pore [16]. 
The electrochemical impedance spectroscopy (EIS) was used to study the voltage-gated channel formation by alamethicin incorporated in the mercury-supported tethered lipid bilayer [17]. Most recently, Su et al. [18] performed impedance spectroscopy studies on alamethicin incorporated into DPhPC bilayers supported at gold electrode surfaces and in tethered bilayer lipid membranes [19]. They supplemented the EIS result with parallel photon polarization modulation infrared reflection absorption spectroscopy (PM-IRRAS) measurements and were able to provide molecular level interpretation of the conductivity through pores formed by alamethicin molecules.

The direct visualization of alamethicin aggregation into channels was achieved by Pieta et al. [20] using the electrochemical scanning tunneling microscopy EC-STM. The EC-STM images showed that alamethicin molecules form porous nanoclusters where an individual peptide participates in the pore formation of two adjacent channels. Unfortunately, these images were only acquired for monolayer films because the tunneling barrier is too large to allow for STM imaging of a bilayer. Therefore, the structure of the peptide aggregates may be influenced by direct contact with the solid gold support.

The objective of this work is to provide direct visualization of alamethicin aggregation in a floating bilayer (fBLM) consisting of 1,2-dimyristoyl-sn-glycero-3-phosphocholine/1,2dimyristoyl-sn-glycero-3-phosphoglycerol (DMPC/DMPG) (1:1) bilayer supported on a 1-thiol$\beta$-D-glucose modified gold (111) electrode by atomic force microscopy (AFM). The combination of the fBLM architecture with the mixed phospholipid matrix (neutral DMPC and negatively charged DMPG) are used to better represent the membrane environment of a Gram-positive bacteria for alamethicin incorporation and channel formation. The modification of the gold surface with a monolayer of 1 - thiol- $\beta$-D-glucose separates the DMPC/DMPG membrane from the gold surface by creating water-rich spacer region [21]. The AFM images of alamethicin aggregates are complemented by EIS measurements of conductivity through alamethicin transmembrane pore. This approach provides unique understanding of the nature of alamethicin antimicrobial activity and insight into the design of novel antimicrobial peptides to combat the growing number of antibiotic resistant bacteria.

\section{Experimental Section}


2.1 Chemicals and Solutions. 1,2-Dimyristoyl-sn-glycero-3-phosphocholine (DMPC) and 1,2dimyristoyl-sn-glycero-3-phosphoglycerol (DMPG) were purchased from Avanti Polar Lipids (99\% pure; Alabaster, AL, US). Alamethicin (Alm) and 1-thio- $\beta$-D-glucose (Tg) were purchased from Sigma-Aldrich (St. Louis, MO, US). These molecules were used as purchased without further purification. HPLC-grade chloroform was purchased from Sigma-Aldrich (St. Louis, MO, US) and used to prepare all vesicle solutions. Sodium fluoride powder, 99\% purity (SigmaAldrich, St. Louis, MO, US), was cleaned in a UV-ozone chamber (Jelight, Irive, CA, US) for 20 min to oxidize and remove any organic impurities prior to use. The sodium fluoride electrolyte solutions were prepared by dissolving the pre-cleaned powder in Milli-Q UV plus ultra-pure water, resistivity $18.2 \mathrm{M} \Omega \mathrm{cm}$, (EMD Millipore, Billerica, MA, US) to give a final concentration of $100 \mathrm{mM}$ and $1 \mathrm{mM}$ for the EIS and AFM experiments, respectively.

2.2 Sample Preparation and Bilayer formation. All glassware was cleaned in a hot mixed acid bath (1-part $\mathrm{HNO}_{3}$ : 3-parts $\mathrm{H}_{2} \mathrm{SO}_{4}$ ) for 60 min and then thoroughly rinsed and soaked in Milli-Q water for 3-4 hrs. The Teflon pieces were soaked in a Piranha solution (1-part $\mathrm{H}_{2} \mathrm{O}_{2}$ : 3parts $\mathrm{H}_{2} \mathrm{SO}_{4}$ ) and then rinsed with Milli-Q ultra-pure water.

The gold substrates for the AFM studies were produced in the AXXIS vacuum system (Kurt J. Lesker, PA, US) by magnetron sputtering technique (Torus, 3" dia., DC). A 3-5 nm titanium adhesion layers were first deposited onto a clean, standard glass microscope slides. Next 200-nm of gold was deposited onto the titanium adhesion layer to create gold substrates that are highly uniform and stable. The gold-coated glass slides were then annealed in a muffle furnace at $675{ }^{\circ} \mathrm{C}$ for 70 seconds to produce large and well-ordered gold crystallites with (111) facets preferentially oriented in parallel to the electrode surface $[21,22]$. The gold slides were then immersed into a $2 \mathrm{mM}$ aqueous solution of $\mathrm{Tg}$ for $20 \mathrm{~h}$ to ensure that a uniform self-assembled monolayer (SAM) is deposited on the gold surface. The Tg SAM is highly hydrophilic and acts as a conditioning layer to assist with vesicle fusion to form model bilayers that better mimics biological conditions.[21] Afterwards, the Tg-modified gold (111) surface was rinsed using copious amounts of water to remove residual $\mathrm{Tg}$ molecules that are physically adsorbed to the surface.

Vesicle solutions were prepared using Barenholz method [23]. Stock solutions of DMPC, DMPG and alamethicin were prepared by dissolving each powder in chloroform to give a final 
concentration of 4.54, 3.6 and $2 \mathrm{mg} / \mathrm{ml}$. The appropriate concentration of each stock solution was then combined in a single test tube and solvent was slowly evaporated by vortexing the mixture under a stream of argon producing a dried film of either DMPC/DMPG (1:1 molar ratio) or DMPC/DMPG in the presence of 10\% Alm (DMPC/DMPG/Alm; 9:9:2 molar ratio). The test tubes containing dried films were placed in a vacuum desiccator for at least $24 \mathrm{~h}$ prior to use to assure that the films were completely free of any residual solvent. The vesicles were then formed in the test tube by adding $1 \mathrm{ml}$ Milli-Q water to the dry lipid film to give a final concentration of $1 \mathrm{mg} / \mathrm{ml}$. The mixture was sonicated for $30 \mathrm{~min}$ at $35{ }^{\circ} \mathrm{C}$ to ensure that vesicles with the appropriate size and distribution were formed in water. Using AFM and the neutron reflectivity techniques, we have previously demonstrated that this procedure leads to the formation of single bilayers at the gold electrode surface [22,24].

The working electrode for electrochemical measurement was a single crystal gold (111) electrode with a surface area of $0.172 \mathrm{~cm}^{2}$, which was prepared in accordance to the procedure described by Richer et al. [25]. The working electrode was flame-annealed with a Bunsen burner, air-dried, and cooled before placing into a beaker containing $2 \mathrm{mM} \mathrm{Tg} \mathrm{SAM} \mathrm{solution} \mathrm{for} 20 \mathrm{~h}$. The modified gold (111) electrode was then removed from the SAM solution and rinsed with sufficient amounts of Milli-Q water. The modified gold slide and modified gold electrode were transferred to a beaker containing the mixed vesicles. The gold slides and gold electrode were incubated for $12 \mathrm{hr}$ to allow for bilayer formation.

2.3 AFM measurements. Dynamic MAC mode AFM images were acquired with an Agilent Technologies 5500 Scanning Probe Microscope (Agilent N9621-13601 MAC III Mode controller, Agilent Technologies, CA, US) in a $1 \mathrm{mM} \mathrm{NaF}$ solution. All images were recorded at $21 \pm 0.5^{\circ} \mathrm{C}$ using type VII MAC cantilevers (Keysight Technologies, ON, CA) with nominal spring constant of $0.14 \mathrm{~N} / \mathrm{m}$ and resonance frequency of $8-10 \mathrm{kHz}$ in solution. The AFM scanner (model N9520A-US07480132) was calibrated using an AFM standard sample with a pitch of $145 \mathrm{~nm}$ pitch (Ted Pella Inc, CA, US) prior to imaging. The topography, amplitude and phase images were simultaneously recorded at a scan speed between 2.0 to 3.5 lines per second. The system was allowed to stabilize for a minimum of $30 \mathrm{~min}$ before acquiring any AFM images. Data acquisition and analysis were carried out using PicoView 1.49 (Agilent Technologies, ON, CA) and Gwyddion v2.40 (Czech Metrology Institute, Brno, CZ) software, respectively. 
The force versus distance curve measurements were carried out in solution using V-shape silicon nitride cantilevers. The spring constant of the cantilever used in the force-distance curves was $0.06 \mathrm{~N} \mathrm{~m}^{-1}$. It was determined by thermal tune method prior to the experiment. Force spectroscopy curves were recorded by measuring the deflection of the cantilever versus the position of the Z-piezo of the scanner. The time required to record a single force-distance curve was 1.59 seconds where the total distance traveled by the tip was $\sim 400 \mathrm{~nm}$ (approach and retraction). The cantilever deflection versus piezo position curves were converted to forcedistance curves (force vs. tip-substrate distance) using software that was written in-house. For statistical data analysis, at minimum of 125 force measurements were measured. All AFM measurements were performed at the open circuit potential (ocp) $\sim 0.1 \mathrm{~V}$ vs the $\mathrm{Ag} / \mathrm{AgCl}$ electrode.

2.4 Electrochemical impedance spectroscopy. The EIS measurements were carried out in an all-glass three-electrode cell consisting of a gold (111) working electrode, a coiled platinum wire as the counter electrode and a $\mathrm{KCl}$ saturated $\mathrm{Ag} / \mathrm{AgCl}$ reference electrode (sat. $\mathrm{KCl}, 0.197 \mathrm{~V}$ vs. SHE). The electrochemical cell was deaerated by purging with argon for $30 \mathrm{~min}$. The gold (111) crystal face covered by the floating bilayer was horizontally touched to the supporting electrolyte and then slowly raised to attain a hanging meniscus configuration where only the single crystal surface was in contact with the electrolyte solution. To prevent the influx of oxygen, an argon blanket was maintained over the solution throughout the duration of the experiments. EIS was performed using the Solartron SI 1287 electrochemical interface (Ametek Scientific Instruments, Oakridge, TN, US) and Solartron SI 1260 impedance/gain-phase analyzer (Ametek Scientific Instruments, Oakridge, TN, US). EIS spectra were collected in the frequency range from $10^{-2}$ to $10^{3} \mathrm{~Hz}$ at potential $+0.1 \mathrm{~V}$ vs $\mathrm{Ag} / \mathrm{AgCl}$ electrode (equal to the open circuit potential) and excitation amplitude of $0.005 \mathrm{~V}$. Data processing of the EIS results was conducted using the ZView software (Scribner Associates Inc., Brno, CZ).

\section{Results}

\subsection{Characterization of the bilayer properties.}


3.1.1 Surface Topography: Figure 1 (a) and (b) depict the surface topography and phase AFM images of DMPC/DMPG bilayers in the absence of alamethicin, respectively. The AFM topography image shows that the Tg-modified gold electrode is uniformly covered by a corrugated film with a periodicity of $6.5 \pm 1 \mathrm{~nm}$ and mean square roughness of $0.66 \mathrm{~nm}$. This characteristic structure has been previously observed by Li et al. [22] for a pure DMPC bilayers deposited on bare gold (111) electrode surfaces using vesicle fusion. In these studies, the corrugated film had a periodicity of $5.5 \pm 0.5 \mathrm{~nm}$ and roughness of $0.5 \pm 0.1 \mathrm{~nm}$. Additionally, a corrugated film structure was also observed for mixed GM1/DMPC/cholesterol bilayers supported on the Tg-modified gold (111) electrode [26]. The corrugation of this bilayer had periodicity of $7 \pm 1 \mathrm{~nm}$ and amplitude of $0.3 \pm 0.1 \mathrm{~nm}$. The formation of the corrugated structure is caused by the elastic stress induced by spontaneous curvature of the lipid molecules. Phospholipid bilayers are known to form a ripple phase, which is a transition from an ordered gel state to a disordered liquid-crystalline state [27-30]. In multiple bilayers, the ripple phase has a periodicity of 12 to $16 \mathrm{~nm}$. The periodicity of the bilayers assembled on the gold electrode range from 5.5 to $7 \mathrm{~nm}$ depending on the lipid composition. This difference in periodicity is due to
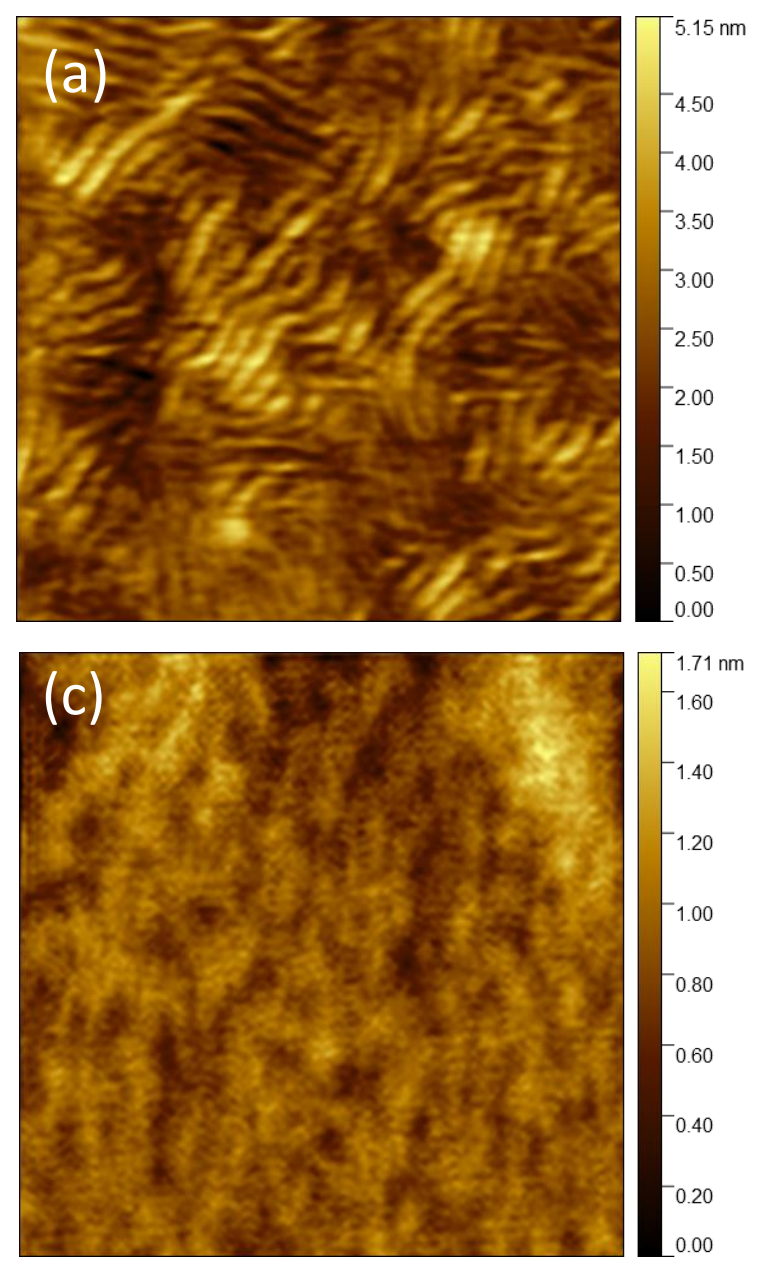
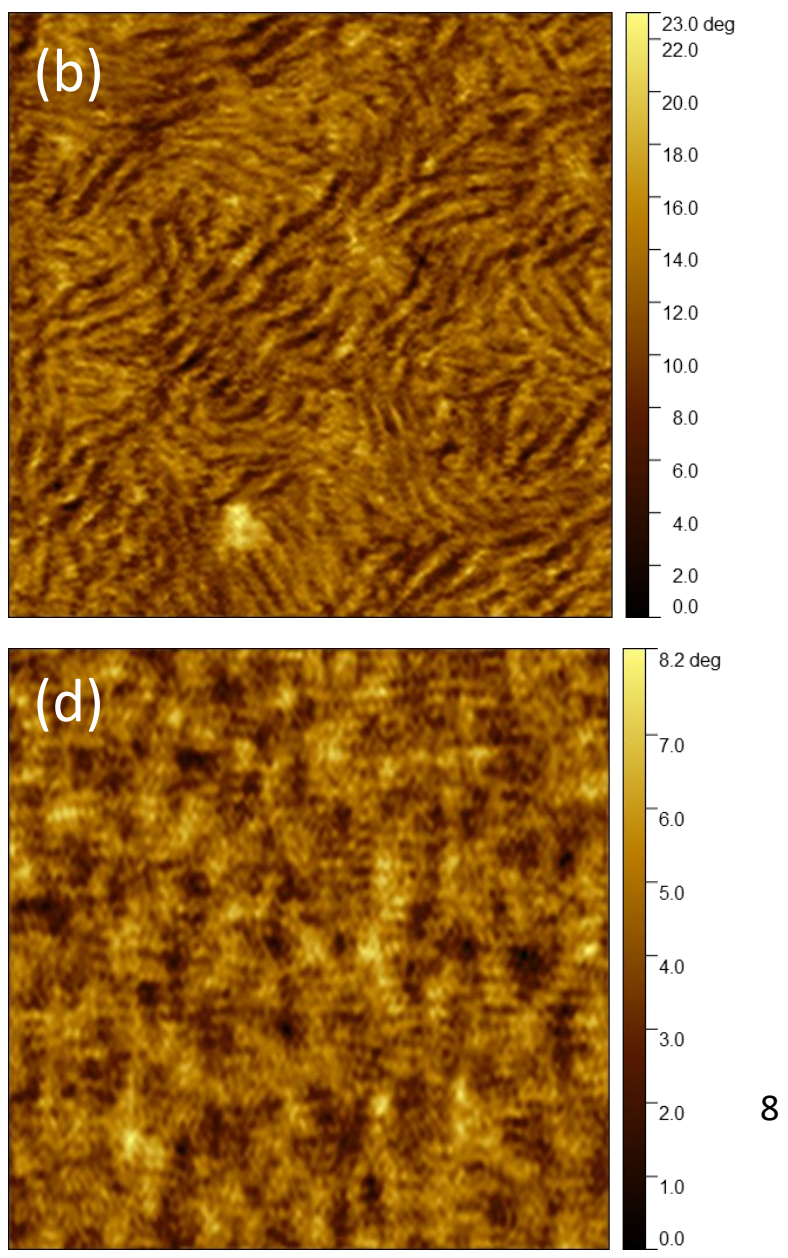
Figure 1. AFM (a) topography and (b) phase images of the pure DMPC/DMPG (1:1) bilayer and AFM (c) topography and (d) phase images of DMPC/DMPG/Alm (9:9:2) bilayer formed on the Tg-modified gold (111) electrode surface by vesicle fusion. All four images were obtained using a scan size of 200 X $200 \mathrm{~nm}$

influence of the substrate [22]. The phase image of the DMPC/DMPG bilayer on Tg-modified gold electrode, presented in Figure 1b, shows no segregation or island formation suggesting that the two different phospholipids are miscible. As a result, the DMPG molecules are evenly distributed within the bilayer ensuring a uniform charge distribution at the electrode surface.

Figure 1 (c) and (d) present the topography and phase images of the bilayer formed at the modified gold (111) electrode surface after fusion of DMPC/DMPG vesicles consisting of alamethicin at a molar concentration of $10 \%$. The corrugated film structure of the pure DMPC/DMPG bilayer, shown in Figure 1 (a), disappears when 10\% alamethicin is incorporated into the DMPC/DMPG vesicles prior to vesicle fusion. This result is consistent with the grazingincidence small-angle X-ray scattering experiment by Salditt et al. [13], who demonstrated that the ripple phase is absent in the DMPC bilayer with alamethicin at the $(1 / 25) \mathrm{L} / \mathrm{P}$ ratio. A similar change in the bilayer structure was also observed by Chen et al. [31] where the corrugations in the DMPC bilayer were removed by addition of cholesterol. The molecular structure of the bilayer presented in Figures 1(c) and (d) will be discussed later on in further detail.

3.1.2 Nanomechanical properties: The force spectroscopy was employed to measure the thickness of the deposited films and confirm that the adsorbed film adopts a bilayer structure. Figures 2 (a) and (d) show typical force-distance curves for the DMPC/DMPG film adsorbed onto the Tg-modified gold (111) surface in the absence and presence of alamethicin recorded during the cantilever approach at a rate of $0.26 \mu \mathrm{m} / \mathrm{s}$. As the tip-sample distance becomes less than $15 \mathrm{~nm}$, the applied force begins to increase due to repulsive forces (primarily coulombic and hydration forces) between the cantilever tip and adsorbed DMPC/DMPG/Alm film. When the separation distance between the tip and sample is $\sim 5 \mathrm{~nm}$, a discontinuity is observed. This is followed by a rapid vertical increase in the cantilever force, which implies that the AFM tip is directly in contact with the gold surface. The discontinuity in the force curve corresponds to a 
characteristic "jump-in" distance, which is related to the thickness of the adsorbed film. These measurements were recorded 125 times at different locations on the gold slide to ensure that the measurements are statistically relevant.

Figures 2 (b) and (e) are histograms displaying the values of the "jump-in" distances from the force-distance curves of the DMPC/DMPG and DMPC/DMPG/Alm films using a bin size $0.5 \mathrm{~nm}$. A Gaussian distribution was applied to the histogram giving a mean "jump-in" distance of $4.2 \pm 0.6 \mathrm{~nm}$ for DMPC/DMPG film and $4.3 \pm 0.9 \mathrm{~nm}$ for the DMPC/DMPG/Alm film. Furthermore, the width of the "jump-in" distances for both films are within the uncertainty of the experiment indicating that Alm has a small effect on the overall film thickness. This result is consistent with X-ray reflectivity measurements performed on highly aligned solid supported multilamellar DMPC/DMPG (1:1) lipid membranes which demonstrated and the thickness of this membrane was $\sim 4.8 \mathrm{~nm}$ and weakly dependened on the alamethicin content [32]. One major difference is that the spread of the "jump-in" distances is much larger for the Alm incorporated film suggesting that the film thickness changes more significantly across different regions of the

\section{DMPC/DMPG}
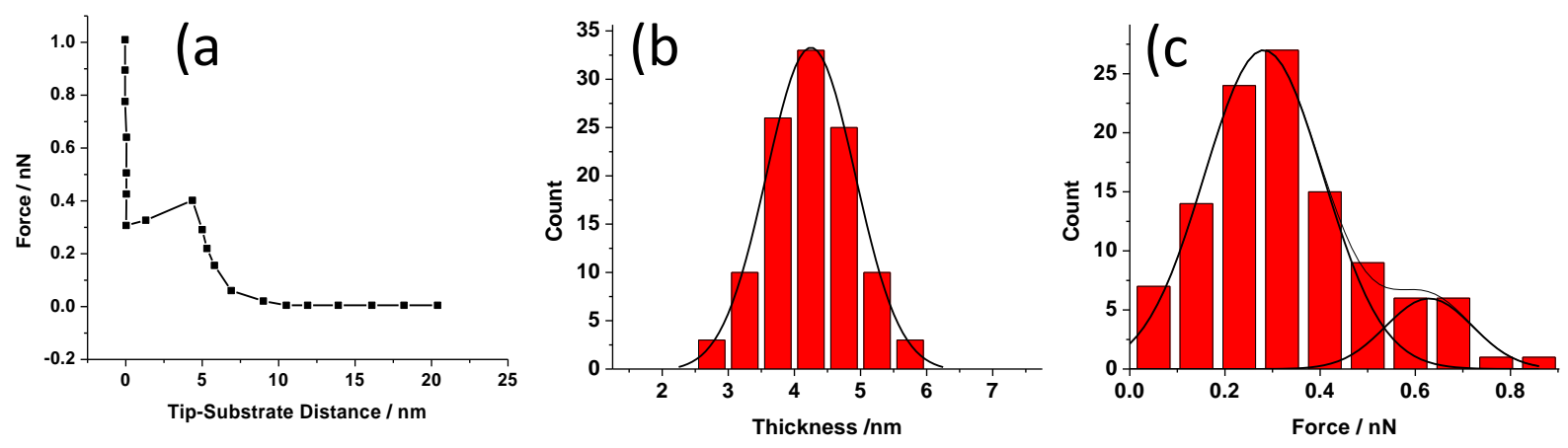

DMPC/DMPG/AIm
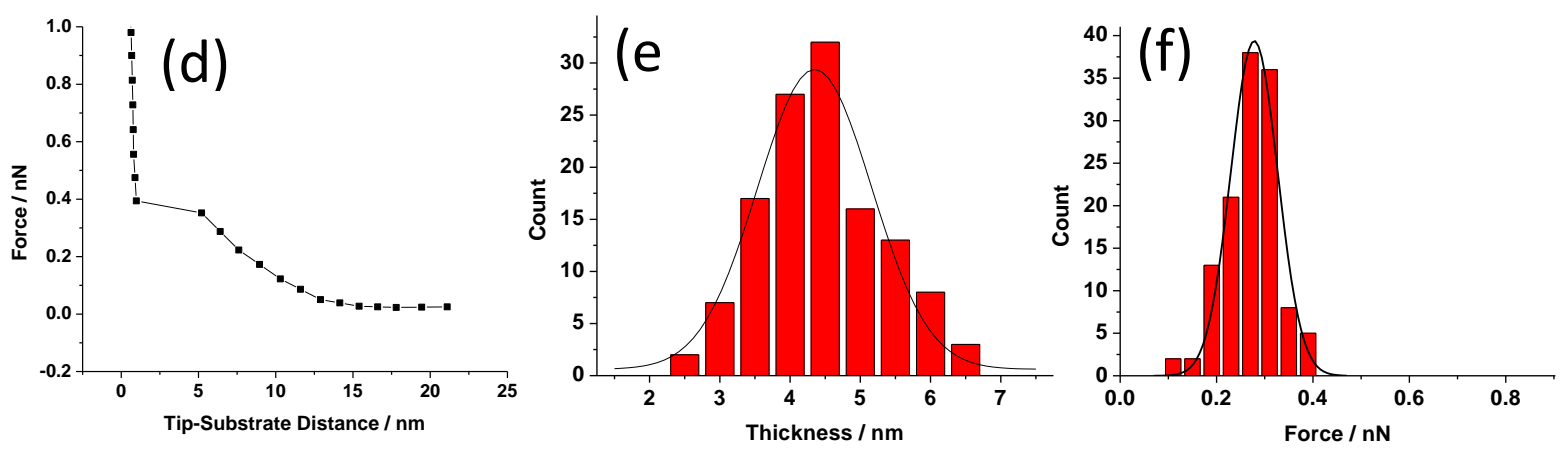
Figure 2. Typical force-distance curves of DMPC/DMPG bilayers in absence (a) and presence of 10 mol \% Alm (d) deposited on a Tg-modified gold slide, respectively. Histograms of the measured jump-in width on the force distance curves for the pure DMPC/DMPG (b) and DMPC/DMPG/Alm (e) fBLMs film thickness. Histograms of the corresponding penetration force for the pure (c) and alamethicin-incorporated bilayers (f). The samples were measured in a $1 \mathrm{mM} \mathrm{NaF}$ electrolyte at room temperature.

surface when Alm is added to the phospholipid matrix. Prior to tip penetration, a compressive force applied to the phospholipid surface, results in elastic deformation of the adsorbed film [3336]. According to Dufrene et al. [36], the elastic deformation, $\delta$, produced by the AFM tip under a known loading force can be modeled using the Hertzian formula

$$
\delta=\left(\frac{9 F^{2}}{16 R E^{2}}\right)^{1 / 3}
$$

where $R$ is the radius of the tip curvature $(10 \mathrm{~nm}), F$ is the average penetration force $(0.3 \mathrm{nN})$ and $E^{*}$ is the effective compression modulus $\left(6.6 \times 10^{7} \mathrm{~N} \mathrm{~m}^{-2}\right)$, which is determined from the Young modulus of a bilayer in liquid crystalline state [30,37]. By applying Equation 1 to the collected force data, the elastic deformation of the film by the tip was estimated to be $1.2 \mathrm{~nm}$. As a result, the corrected bilayer thickness of the DMPC/DMPG/Alm bilayer is $5.5 \pm 0.9 \mathrm{~nm}$. This number is consistent with the expected value for a floating phospholipid bilayer confirming that the structure of the DMPC/DMPG/Alm adsorbed film at the gold (111) electrode surface is in fact a bilayer.

Figures 2 (e and f) display histograms of the "jump-in" force for the two bilayers. The jump- in (or bilayer penetration force) provides useful information about the nanomechanical properties of the bilayer and indirectly on the lateral interactions between the membrane molecules [38,39]. In the molecular model [33,34], the penetration force is interpreted as the force at which $N$ elastically compressed molecules acquire sufficient energy to jump out from their equilibrium position within the bilayer and form a hole that is sufficiently large to allow the tip to penetrate the bilayer. It is important to note, that the penetration force is dependent not only on the intrinsic properties of the bilayer being measured, but also on the chemical properties of the AFM tip, cantilever spring constant, radius of the AFM tip and approach velocity of the AFM tip used to acquire the force-distance curve. Therefore, penetration force measurements can only be 
compared for experiments performed at identical conditions. For the DMPC/DMPG bilayer, the average penetration force amounts to $0.32 \pm 0.17 \mathrm{nN}$, while the average force is equal to $0.30 \pm$ $0.10 \mathrm{nN}$ for the DMPC/DMPG/Alm bilayer. The differences are small and suggest that addition of Alm has little effect on nanomechanical properties of the bilayer. These values are significantly lower than the values obtained from our previous measurements for pure DMPC and DMPC/Chol bilayers, which were $1.2 \pm 0.5$ and $1.7 \pm 0.5 \mathrm{nN}$, respectively, determined at similar experimental conditions [22,31]. The pure DMPC and DMPC/Chol bilayers investigated in [22] and [31] were predominantly in the gel state since the experiments were performed at 18 $\pm 1{ }^{\circ} \mathrm{C}$. The lower values of the penetration force observed for the DMPC/DMPG and DMPC/DMPG/Alm bilayers suggest that the latter are in the liquid crystalline state [40]. However, the distribution of penetration forces is much broader for the DMPC/DMPG bilayer. In fact, the distribution of penetration forces appears to be bimodal with a second small maximum observed at $\sim 0.64 \mathrm{nN}$. This behavior may be a result of the stress in the DMPC/DMPG bilayer. In contrast, the penetration force distribution for the DMPC/DMPG/Alm bilayer is very narrow and symmetric implying that the addition of alamethicin improves elastic properties of the mixed bilayer.

3.1.4 Membrane resistivity. To determine the effect of alamethicin on membrane resistivity, EIS measurements were performed using frequencies ranging from $10^{-2}$ to $10^{3} \mathrm{~Hz}$ at a bias potential of $0.1 \mathrm{~V}$ vs. $\mathrm{Ag} / \mathrm{AgCl}$ (open circuit potential). Figure 3 (a) and (b) display the total impedance and corresponding phase angle of the DMPC/DMPG (1:1 mol ratio) bilayers adsorbed at the Tg-modified gold electrode surface in the absence and presence of $10 \%$ alamethicin. The impedance of the phospholipid bilayer without the peptide is larger than that of the bilayer with alamethicin at all studied frequencies. Additionally, the phase angle plot in Figure 3 (b) shows that the incorporation of alamethicin into the bilayer causes a decrease of the phase angle at frequencies below $1 \mathrm{~Hz}$. Valincius et al. have shown that this behavior indicates pore formation by alamethicin molecules in the bilayer $[41,42]$. Since, the phase angle plots do not display a minimum the density of pores or defects must be very low. In that case, the EIS data can be conveniently discussed using a model of a membrane with isolated defects or pores developed by Valincius et al [42]. The impedance of a floating bilayer can be considered to be represented by the generic equivalent circuit Figure 3 (a). The elements of this circuit are $R_{s}$ representing the resistance of the solution, $Z^{\prime}{ }_{\text {mem }}$ representing the membrane, $Z_{\text {def }}$ representing the 
impedance of defects or peptide created pores and $Z_{s u b}$ representing the impedance of the submembrane layer between the membrane and the gold surface. The $Z^{\prime}{ }_{\text {mem }}$ can be represented by a capacitor, $Z_{\text {def }}$ by a resistor, $Z_{\text {sub }}$ depends on the propagation of ions in the submembrane layer radially from the defect or the pore site and parallel to the gold surface. It may be represented by infinite network of resistors and capacitors [42] as schematically shown in Figure 4. The $Z_{\text {sub }}$ has complex properties and its value depends on the defects size and density, resistivity and thickness of the
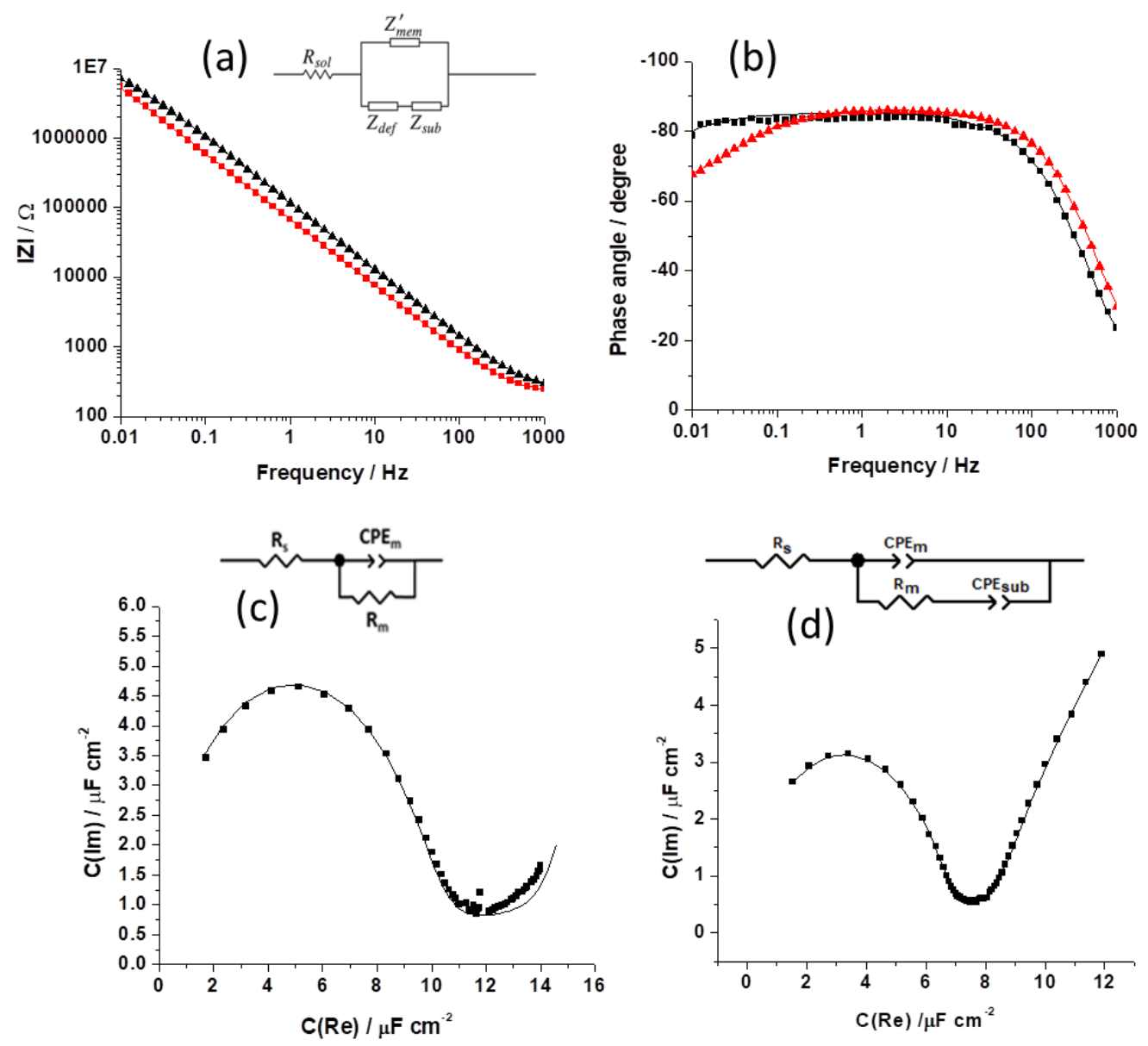

Figure 3 (a) Bode and (b) phase angle plots versus frequency showing the EIS data of the DMPC/DMPG (black) and DMPC/DMPG/10\% Alm (red) bilayers supported at the gold (111) electrode modified with a monolayer of $\beta$-thioglucose. (c) Cole-Cole plot of the EIS data for the DMPC/DMPG bilayer and (d) Cole-Cole plot for the DMPC/DMPG/10\% Alm bilayer. Inset to figure a represents a generic equivalent circuit of the floating bilayer. The solid lines represent fits to the experimental data with the equivalent circuits shown in figures $\mathrm{c}$ and d, respectively. The EIS measurements were performed in $0.1 \mathrm{M} \mathrm{NaF}$ electrolyte at $\mathrm{E}=0.1 \mathrm{~V}$ vs $\mathrm{Ag} / \mathrm{AgCl}$ reference electrode 
submembrane layer and the frequency range used in the EIS measurements [42]. The $Z_{\text {sub }}$ becomes resistive when defects are small, the thickness of the submembrane layer is small and its resistivity is high. The resistive elements of the network become dominant. In contrast, the $Z_{\text {sub }}$ can be approximated by a constant phase element (CPE)

$Z_{C P E}(\omega)=\frac{1}{Q(i \omega)^{\alpha}}$

with $\alpha \sim 0.5$ when the size and the density of defects (pores ) are higher and the submembrane layer becomes more conductive. In equation 2, $\omega$ is the angular frequency, $Q$ is the coefficient of the constant phase element and $\alpha$ is a measure of homogeneity where a value of 1 is given for purely homogeneous films [42].

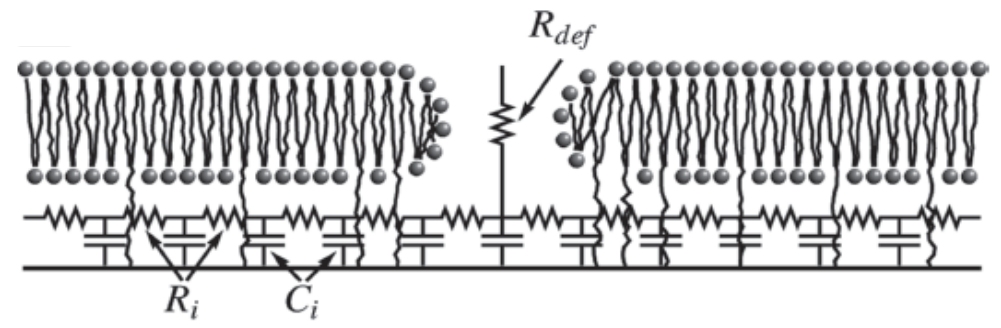

Figure 4 Model of the fBLM with distributed network of $C$ and $R$ elements that represent propagation of ac current in the submembrane layer radially with respect to the defect and parallel to the metal surface; reproduced from ref 41.

The complex nature of impedance of the submembrane ionic reservoir separating the phospholipid layer and the solid support see (Fig. 4) explains the use of $\mathrm{CPE}_{\text {sub }}$ in the equivalent circuit given in Fig. 3 (d).

Figures 3 (c) and (d) show Cole-Cole plots of the DMPC/DMPG and DMPC/DMPG/Alm bilayers, respectively. The solid lines show the resulting data fits by the schematic models presented in the insets of these figures. For the DMPC/DMPG bilayer, the impedance data can be modeled if $Z_{\text {sub }}$ is represented by a resistor. When a CPE was used to represent $Z_{\text {sub }}$ the fit gave coefficient $\alpha<0.1$ a value which has no physical meaning. The fit is very good up to the minimum at $\operatorname{Re} C \sim 12 \mu \mathrm{F} / \mathrm{cm}^{2}$ in the Cole-Cole plot, which corresponds to frequency $10^{-1} \mathrm{~Hz}$. Consequently, the data was only fit using this model in the frequency range between $10^{3}$ and 0.1 Hz. Small deviations are observed in the frequency range between 0.1 and $0.01 \mathrm{~Hz}$ where the 
model representing impedance of the submembrane region may be an oversimplification. For the membrane with $10 \%$ of alamethicin, the $Z_{\text {sub }}$ can be represented by the CPE with $\alpha \sim 0.5$ in the whole frequency range between $10^{3}$ and $10^{-2} \mathrm{~Hz}$, indicating that insertion of alamethicin into the membrane increased the density of defects (pores) and the pore sizes. The numerical values of the fit parameters are reported in Table 1.

Table 1. Numerical values of the elements of the equivalent circuit of gold (111) electrode with DMPC/DMPG bilayers in the absence and presence of $10 \%$ alamethicin in $0.1 \mathrm{M} \mathrm{NaF}$ solution

DMPC/DMPG

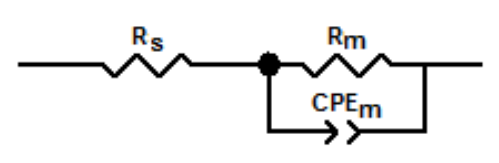

DMPC/DMPG/AIm

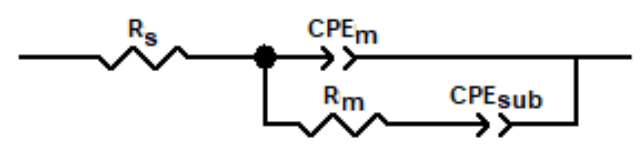

\begin{tabular}{cccccc}
\hline & $\begin{array}{c}\mathrm{R}_{\mathrm{m}} / \mathrm{M} \Omega . \mathrm{cm} \\
\mathbf{2}\end{array}$ & $\begin{array}{c}\mathrm{CPE}_{\mathrm{m}} / \\
\mu \mathrm{F} . \mathrm{cm}^{-2} \cdot \mathrm{s}^{\alpha-1}\end{array}$ & $\boldsymbol{\alpha}_{\mathrm{m}}$ & $\begin{array}{c}\mathrm{CPE}_{\text {sub }} / \\
\mu \mathrm{F} . \mathrm{cm}^{-2} \cdot \mathbf{s}^{\alpha-1}\end{array}$ & $\boldsymbol{\alpha}_{\text {sub }}$ \\
\hline $\begin{array}{c}\text { DMPC/D } \\
\text { MPG }\end{array}$ & $9.4 \pm 0.4$ & $12.5 \pm 2.6$ & $\begin{array}{c}0.948 \pm 0.0 \\
08\end{array}$ & - & - \\
DMPC/D & $1.4 \pm 0.4$ & $8.4 \pm 1.4$ & $0.962 \pm 0.0$ & $2.4 \pm 0.2$ & $0.576 \pm 0.0$ \\
MPG/AIm & & & 02 & & 25 \\
\hline
\end{tabular}

The membrane resistance for the alamethicin-free bilayer has a value of $9.4 \pm 0.4 \mathrm{M} \Omega$ $\mathrm{cm}^{2}$ and decreases considerably to a value of $1.4 \pm 0.4 \mathrm{M} \Omega \mathrm{cm}^{2}$. This indicates that the alamethicin peptides are incorporating into the DMPC/DMPG bilayer and not simply adsorbing to the external surface of the bilayer, which would lead to an increase in membrane resistance. The $\alpha$ values are $\sim 0.95$ for the two bilayers, which implies that both membranes are quite 
homogeneous. The membrane capacitance is $\sim 10 \pm 2 \mu \mathrm{F} \mathrm{cm}^{-2}$ and within experimental error does not change with addition of alamethicin. A similar value of the membrane capacitance was observed by Madrid and Horswell [43] for bilayers of negatively charged dimyristoyl phosphatidylserine (DMPS) supported on gold (111) surfaces. They attributed the high value of the capacitance to high water content within the bilayer resulting from the strong hydration of the negatively charged lipid head groups. The value of $\alpha \sim 0.5$ for the $\mathrm{CPE}_{\text {sub }}$ suggests that $Z_{\text {sub }}$ is controlled by the distributed network of $C$ and $R$ elements that represent propagation of ac current in the submembrane layer [41]. All these results indicate that the electrolyte ions translocate across assembled DMPC/DMPG/Alm bilayer more easily than across the pure DMPC/DMPG bilayers suggesting that alamethicin is either forming defects or pores in the DMPC/DMPG film..

\subsection{Visualization of a pore formed by alamethicin in the DMPC/DMPG bilayer}

To determine whether the defects in the DMPC/DMPG/Alm film were a result of ion pore formation or bilayer disruption, high resolution AFM images were recorded. Figure 5 displays a typical high resolution (100 nm X $100 \mathrm{~nm}$ ) AFM topography image of DMPC/DMPG
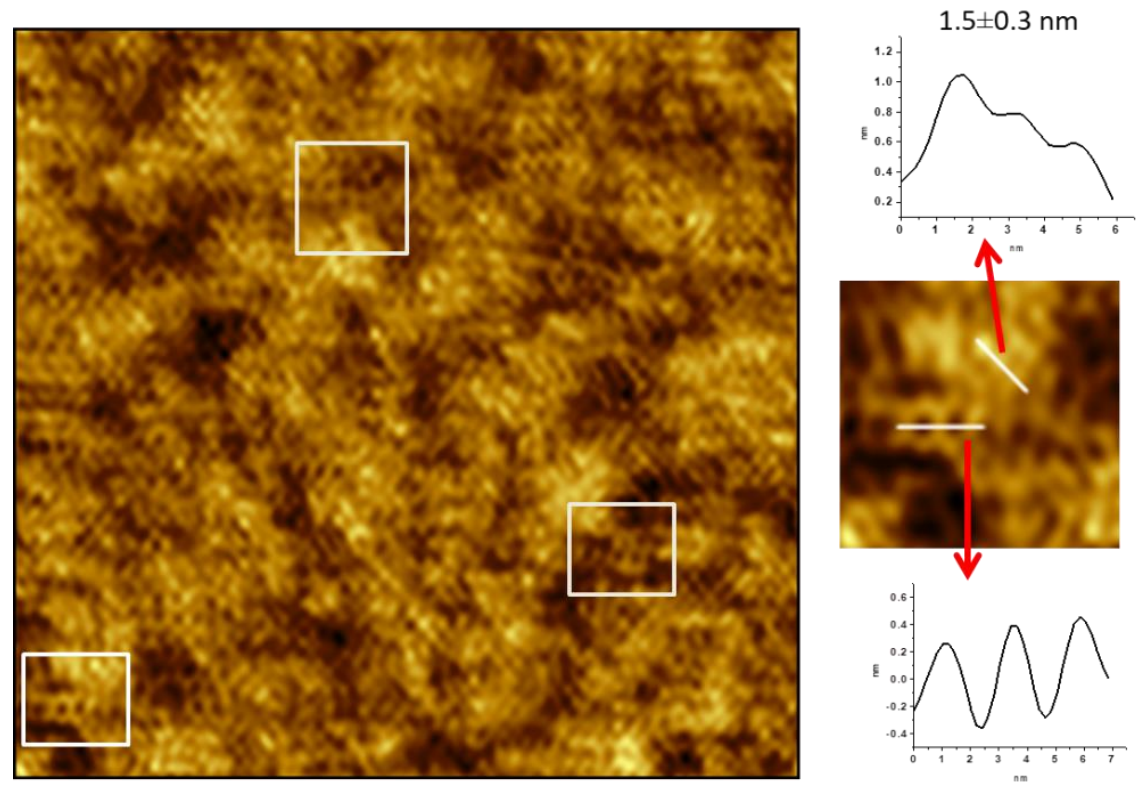

$2.3 \pm 0.3 \mathrm{~nm}$

Figure 5. (a) High resolution AFM topography image (size $100 \mathrm{~nm} \times 100 \mathrm{~nm}$ ) of the DMPC/DMPG/Alm (10\%) bilayer formed on the Tg-modified gold (111) electrode surface by 
vesicle fusion. The images on right show a zoomed in fragment taken from the bottom left corner of the image (size $20 \times 20 \mathrm{~nm}$ ) and cross sections of two different molecular packing observed in this fragment.

bilayer containing $10 \%$ Alm. The contrast reveals irregular molecular clusters resembling rows of phospholipid molecules and hexagonal-like structures suggesting presence of pores within the liquid-crystalline phospholipid matrix. An enlarged region, $20 \mathrm{~nm} \mathrm{X} 20 \mathrm{~nm}$, from the bottom left corner of Figure 5 (a) is presented in Figure 5 (b). This zoomed image shows two distinct molecular clusters where one corresponds to the tightly packed liquid-crystalline DMPC/DMPG molecules and the other corresponds to the pore structures formed by the inclusion of $10 \% \mathrm{Alm}$.

The cross-section profile above the image Figure 5 (b) shows the distance between regular bright spots in the cluster of DMPC/DMPG molecules. Similar molecular rows could be seen in many parts of the image in Figure 5. Numerous cross sections of these bright spots in rows were measured giving an average periodicity of $1.5 \pm 0.3 \mathrm{~nm}$. In the STM images of the compact phospholipid monolayers deposited onto the gold (111) surface with the head groups in contact with the gold surface and terminal methyl groups of acyl chains directed to the STM tip, Sek et al. [44] and Pieta et al. [20] observed the distance between adjacent lipid chains to be 0.5 $\pm 0.1 \mathrm{~nm}$ the distance between the centroid of neighboring molecules of $0.9 \pm 0.2 \mathrm{~nm}$. The contrast in AFM images in Figure 5 corresponds to the interactions between the AFM tip and the head groups of the bilayer. Therefore, the periodicity $1.5 \pm 0.3 \mathrm{~nm}$ corresponds to the distance between polar heads of hydrated phospholipid molecules, expected to be somewhat larger that the distance between closely packed lipid chains. Specifically, the bilayer imaged in Figure 5 (a) was formed by vesicles fusion while the monolayers investigated in $[20,44]$ were transferred in a highly compressed state from the air-solution interface using the Langmuir-Blodgett (LB) technique. Li et al. [22] demonstrated that lipid molecules are more tilted in bilayers formed by vesicles fusion than by the LB method. Furthermore, PM-IRRAS work by Garcia et al. [45] showed that the molecules in the inner leaflet (i.e. in contact with the gold surface) adopted an average tilt angle of $25^{\circ}$ while the phospholipid molecules in the outer leaflet assumed an average angle of $35^{\circ}$. An increase in tilt angle by $\sim 10^{\circ}$ would result in an increase in the cross section of lateral footprint of the phospholipid molecule by $\sim 0.5 \mathrm{~nm}$.

The cross-sectional measurement below Figure 5 (b) shows a line profile of the pore-rich domain. Similar pore-rich domains could also be seen in other areas of Figure 5 (a). The average diameter of the pores obtained from numerous cross-sectional measurements gave a value of 2.3 
$\pm 0.3 \mathrm{~nm}$, which was determined from the analysis of the peak-to-peak height. This number is in a good agreement with the previous STM data obtained by Pieta [20] for alamethicin in a monolayer of phospholipids and molecular dynamic simulations for barrel-stave pore formed by alamethicin molecules [46,47].
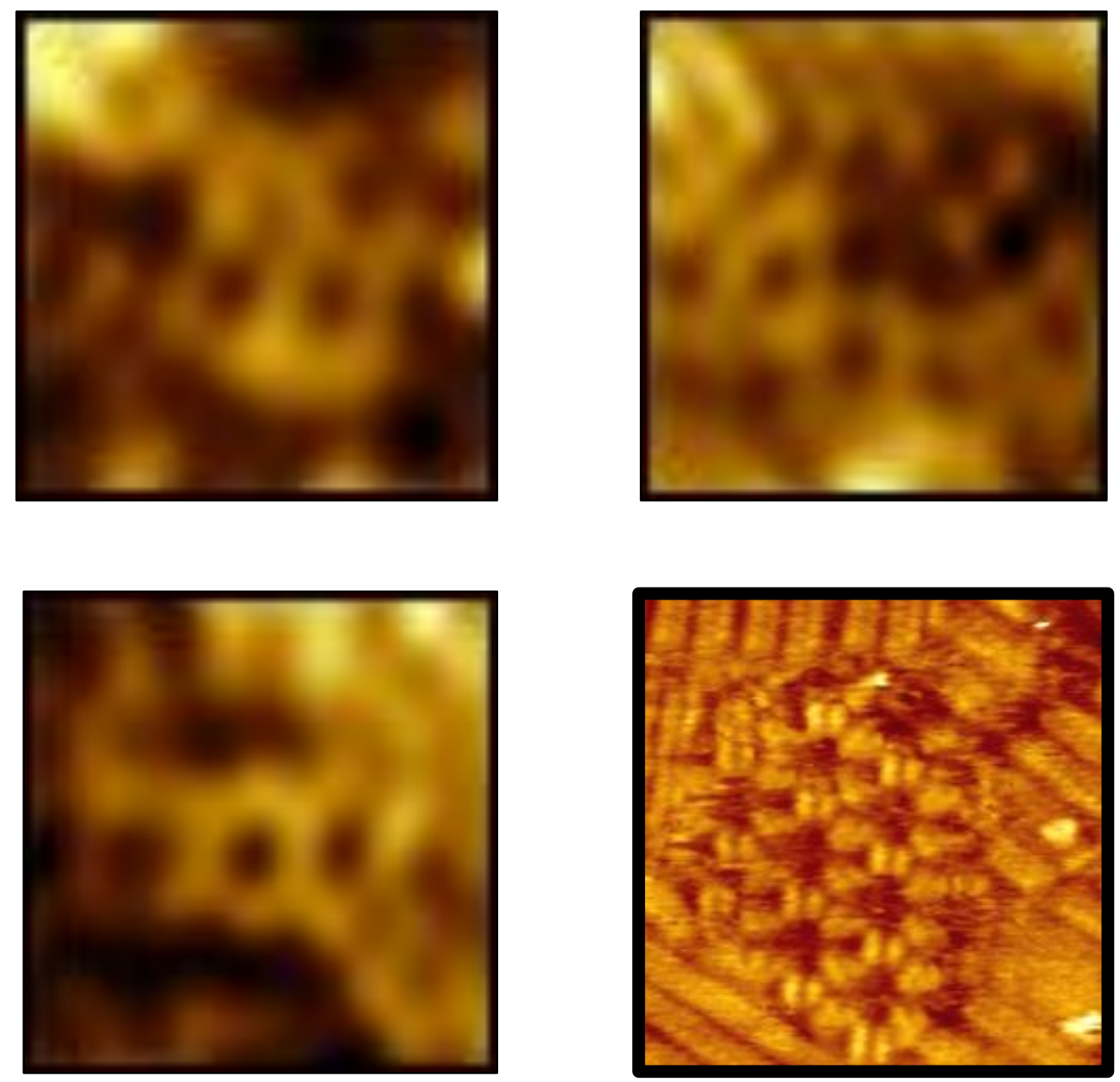

Figure 6. Comparison of three zoomed $(12 \times 12 \mathrm{~nm})$ regions from the AFM displayed in Figure 4 (each denoted by a white frame) with the STM image of alamethicin aggregates in a mixed DMPC/egg PG monolayer taken from the paper by Pieta et al [20].

Figure 6 compares pores from three regions of Figure 5 (marked with white frames) to a cluster of alamethicin molecules observed by Pieta et al. [20] in the STM image of the 
monolayer of phospholipid molecules. The AFM images show that the pores form nanoclusters of different size dimensions; however, the alamethicin clusters in the AFM and STM images are strikingly similar in appearance and distribution. Although the resolution of AFM images is lower than that of the STM image, the comparison of these images shows that hexagonal clusters of molecules are formed not only in the monolayer, but also in the floating bilayer. For comparison with the STM image the clusters in Figure 6 have comparable sizes. Additionally,
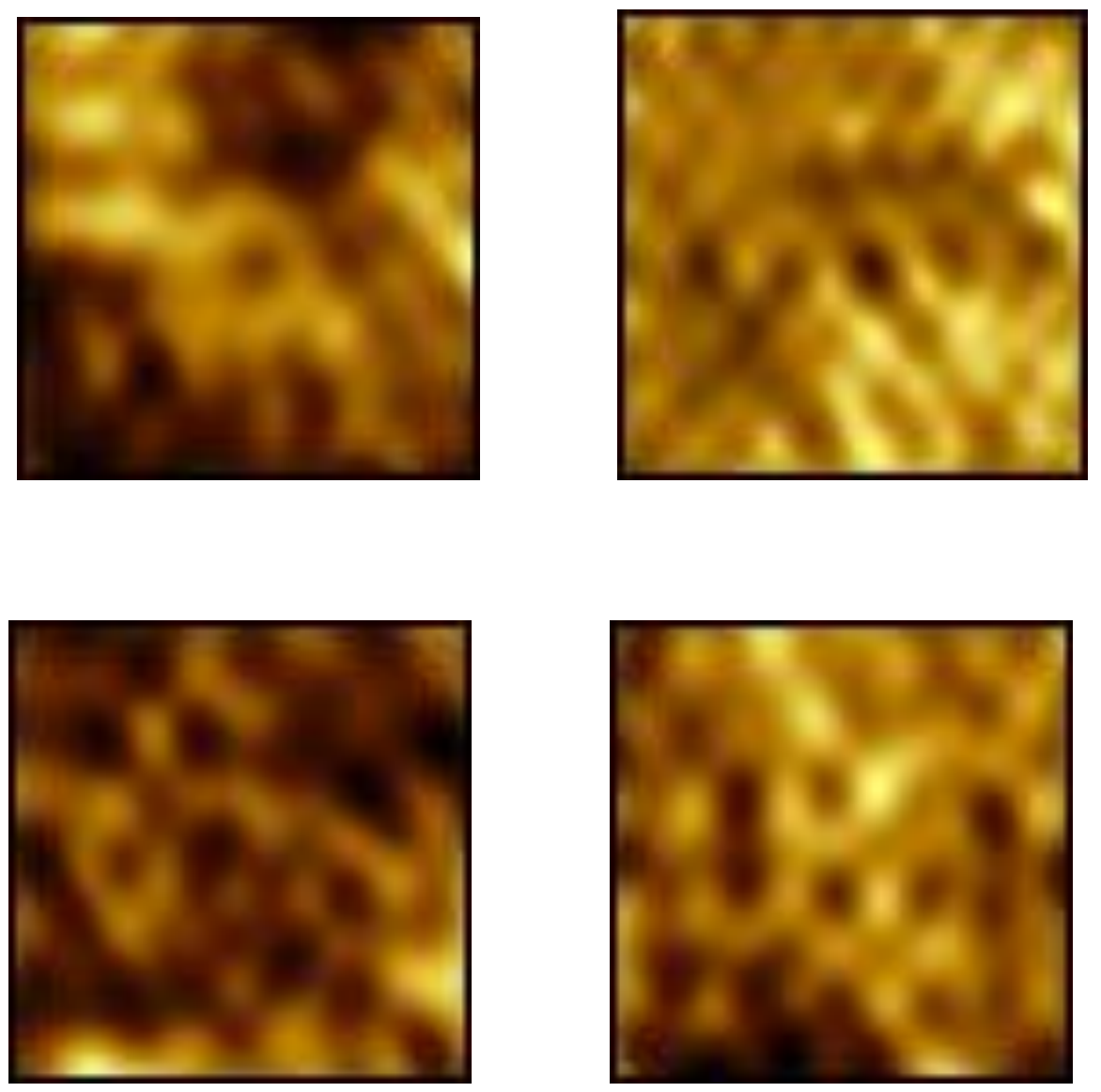

Figure 7. Four zoomed $(12 \times 12 \mathrm{~nm})$ regions from the AFM image in Figure 4 that illustrate that the alamethicin forms clusters with variable number of pores.

images in Figure 7 demonstrate that alamethicin molecules are forming nanoclusters of variable dimensions in the bilayer and hence provide multiple pores for ionic transport across the bilayer. The present study confirms a tendency of alamethicin molecules to form nanoclusters observed previously in the STM studies [20]. However, the STM studies were performed on a monolayer in direct contact with the metal surface, which implies that the cluster formation could be 
templated by that surface. The present study demonstrates that cluster formation is also present in a floating bilayer separated from the metal by a hydrated layer. This unique information confirms that there is a natural tendency for alamethicin peptides to insert and form hexagonal pores in phospholipid bilayers, which is described by the classic barrel stave model, and furthermore, there is a tendency for these to assemble into clusters of different sizes, which explains the conductance behavior previously observed in free-standing black lipid membranes [6].

\section{Conclusions}

The DMPC/DMPG bilayer floating on the gold surface is a good model to study pore formation by peptides. The adsorbed bilayer is homogeneous and separated from the metal by a film of hydrophilic thioglucose. The nanomechanical properties of this bilayer probed by AFM demonstrated that the film has the expected thickness $5.5 \pm 0.9 \mathrm{~nm}$ and that the molecules are present in the liquid crystalline state. The addition of alamethicin improves homogeneity of the bilayer, but has a minor influence on the overall thickness and elasticity of the bilayer. EIS measurements revealed that insertion of alamethicin into the membrane significantly reduces its resistivity indicating that the peptide aggregates in the membrane and forms ion conducting pores. The presence of the pores was revealed by high resolution AFM images, which show that alamethicin forms porous nanoclusteres with an average pore diameter of $2.3 \pm 0.3 \mathrm{~nm}$. The dimension of the pore diameter is consistent with the barrel-stave model of alamethicin aggregates predicted by MD calculations [48,49]. This is a unique visualization of the barrelstave model and provides direct validation of the predictions based on MD calculations. The images show that there is a broad distribution of nanoclusters of the pores in the bilayer matrix. Formation of nanoclusters by alamethicin molecules has been postulated previously by neutron and X-ray in plane scattering $[12,13,46]$. However, neither neutron nor X-ray scattering techniques have sufficient resolution to determine the nanocluster shape and size. The broad distribution of their sizes explains why multiple conductivity states are observed for alamethicin incorporated in free-standing BLMs.[6] These results provide new insights and contribute to our understanding about the nature and mechanism of the antimicrobial activity by amphiphatic peptides. 


\section{Acknowledgements}

This work was supported by a Discovery grant from Natural Sciences and Engineering Council of Canada RG-03958.

\section{Literature}

[1] B. Leitgeb, A. Szekeres, L. Manczinger, C. Vágvölgyi, L. Kredics, The history of Alamethicin: A review of the most extensively studied peptaibol, Chem. Biodivers. 4 (2007) 1027-1051. doi:10.1002/cbdv.200790095.

[2] G. Andrew Woolley, B.A. Wallace, Model ion channels: Gramicidin and alamethicin, J. Membr. Biol. 129 (1992) 109-136. doi:10.1007/BF00219508.

[3] R.O. Fox, F.M. Richards, A voltage-gated ion channel model inferred from the crystal structure of alamethicin at 1.5-A resolution., Nature. 300 (1982) 325-330. doi:10.2210/pdb1amt/pdb.

[4] K. He, S.J. Ludtke, W.T. Heller, H.W. Huang, Mechanism of alamethicin insertion into lipid bilayers., Biophys. J. 71 (1996) 2669-79. doi:10.1016/S0006-3495(96)79458-4.

[5] H.W. Huang, Y. Wu, Lipid-alamethicin interactions influence alamethicin orientation., Biophys. J. 60 (1991) 1079-1087. doi:10.1016/S0006-3495(91)82144-0.

[6] M.S. Sansom, Alamethicin and related peptaibols--model ion channels., Eur. Biophys. J. 22 (1993) 105-24. doi:10.1007/BF00196915.

[7] M.K. Mathew, P. Balaram, A helix dipole model for alamethicin and related transmembrane channels, FEBS Lett. 157 (1983) 1-5. doi:10.1016/0014-5793(83)811053.

[8] M. Eisenberg, J.E. Hall, C.A. Mead, The nature of the voltage-dependent conductance induced by alamethicin in black lipid membranes, J. Membr. Biol. 14 (1973) 143-176. doi:10.1007/BF01868075.

[9] I. Vodyanoy, J.E. Hall, T.M. Balasubramanian, Alamethicin-induced current-voltage curve asymmetry in lipid bilayers, Biophys. J. 42 (1983) 71-82. doi:10.1016/S00063495(83)84370-7.

[10] R. Guidelli, L. Becucci, Mechanism of voltage-gated channel formation in lipid membranes, Biochim. Biophys. Acta - Biomembr. 1858 (2016) 748-755. doi:10.1016/j.bbamem.2015.12.035.

[11] J. Pan, S. Tristram-Nagle, J.F. Nagle, Alamethicin aggregation in lipid membranes, J. Membr. Biol. 231 (2009) 11-27. doi:10.1007/s00232-009-9199-8.

[12] K. He, S.J. Ludtke, D.L. Worcester, H.W. Huang, Neutron scattering in the plane of membranes: structure of alamethicin pores., Biophys. J. 70 (1996) 2659-66. doi:10.1016/S0006-3495(96)79835-1.

[13] T. Salditt, C. Li, A. Spaar, Structure of antimicrobial peptides and lipid membranes probed by interface-sensitive X-ray scattering, Biochim. Biophys. Acta - Biomembr. 1758 (2006) 1483-1498. doi:10.1016/j.bbamem.2006.08.002.

[14] S. Ye, H. Li, F. Wei, J. Jasensky, A.P. Boughton, P. Yang, Z. Chen, Observing a model ion channel gating action in model cell membranes in real time in situ: Membrane potential change induced alamethicin orientation change, J. Am. Chem. Soc. 134 (2012) 6237-6243. doi:10.1021/ja2110784. 
[15] P. Yang, F.G. Wu, Z. Chen, Lipid fluid-gel phase transition induced alamethicin orientational change probed by sum frequency generation vibrational spectroscopy, J. Phys. Chem. C. 117 (2013) 17039-17049. doi:10.1021/jp4047215.

[16] K.F. Wang, R. Nagarajan, T.A. Camesano, Antimicrobial peptide alamethicin insertion into lipid bilayer: A QCM-D exploration, Colloids Surfaces B Biointerfaces. 116 (2014) 472-481. doi:10.1016/j.colsurfb.2014.01.036.

[17] L. Becucci, R. Guidelli, C. Peggion, C. Toniolo, M.R. Moncelli, Incorporation of channelforming peptides in a Hg-supported lipid bilayer, J. Electroanal. Chem. 576 (2005) 121128. doi:10.1016/j.jelechem.2004.09.032.

[18] Z. Su, M. Shodiev, J. Jay Leitch, F. Abbasi, J. Lipkowski, In situ electrochemical and PMIRRAS studies of alamethicin ion channel formation in model phospholipid bilayers, J. Electroanal. Chem. (2017). doi:10.1016/j.jelechem.2017.10.042.

[19] Z. Su, J.J. Leitch, F. Abbasi, R.J. Faragher, A.L. Schwan, EIS and PM-IRRAS studies of alamethicin ion channels in a tethered lipid, J. Electroanal. Chem. (2017) 0-1. doi:10.1016/j.jelechem.2017.12.039.

[20] P. Pieta, J. Mirza, J. Lipkowski, R.W. Murray, Direct visualization of the alamethicin pore formed in a planar phospholipid matrix., Proc. Natl. Acad. Sci. U. S. A. 109 (2012) 21223-7. doi:10.1073/pnas.1201559110.

[21] A.H. Kycia, S. Sek, Z. Su, A.R. Merrill, J. Lipkowski, Electrochemical and STM studies of 1-thio- $\beta$-d-glucose self-assembled on a Au(111) electrode surface, Langmuir. 27 (2011) 13383-13389. doi:10.1021/la202978d.

[22] M. Li, M. Chen, E. Sheepwash, C. L. Brosseau, H. Li, B. Pettinger, H. Gruler, J. Lipkowski, AFM Studies of Solid - Supported Lipid Bilayers formed at a Au ( 111 ) Electrode Surface Using Vesicle Fusion and a Combination of Langmuir - Blodgett and Langmuir - Schaefer Techniques., . Langmuir. 24 (2008) 10313-10323.

[23] Y. Barenholz, D. Gibbes, B.J. Litman, J. Goll, T.E. Thompson, F.D. Carlson, A Simple Method for the Preparation of Homogeneous Phospholipid Vesicles, Biochemistry. 16 (1977) 2806-2810. doi:10.1021/bi00631a035.

[24] I. Burgess, M. Li, S.L. Horswell, G. Szymanski, J. Lipkowski, J. Majewski, S. Satija, Electric Field-Driven Transformations of a Supported Model Biological Membrane-An Electrochemical and Neutron Reflectivity Study, Biophys. J. 86 (2004) 1763-1776. doi:10.1016/S0006-3495(04)74244-7.

[25] J. Richer, Measurement of Physical Adsorption of Neutral Organic Species at Solid Electrodes, J. Electrochem. Soc. 133 (1986) 121. doi:10.1149/1.2108505.

[26] A.H. Kycia, J. Wang, A.R. Merrill, J. Lipkowski, Atomic force microscopy studies of a floating-bilayer lipid membrane on a $\mathrm{Au}(111)$ surface modified with a hydrophilic monolayer, Langmuir. 27 (2011) 10867-10877. doi:10.1021/la2016269.

[27] K. Sengupta, V.A. Raghunathan, J. Katsaras, Structure of the ripple phase in chiral and racemic dimyristoylphosphatidylcholine multibilayers, Phys. Rev. E. 59 (1999) 24552457. doi:10.1103/PhysRevE.59.2455.

[28] W.J. Sun, S. Tristram-Nagle, R.M. Suter, J.F. Nagle, Structure of the ripple phase in lecithin bilayers., Proc. Natl. Acad. Sci. U. S. A. 93 (1996) 7008-7012. doi:10.1073/pnas.93.14.7008.

[29] M.J. Janiak, D.M. Small, G.G. Shipley, Nature of the Thermal Pretransition of Synthetic Phospholipids: Dimyristoyl- and Dipalmitoyllecithin, Biochemistry. 15 (1976) 45754580. doi:10.1021/bi00666a005. 
[30] D. Needham, E. Evans, Structure and Mechanical Properties of Giant Lipid (DMPC) Vesicle Bilayers From $20^{\circ} \mathrm{C}$ Below To $10{ }^{\circ} \mathrm{C}$ Above The Liquid Crystal-crystalline Phase Transition At $24^{\circ} \mathrm{c}$, Biochemistry. 27 (1988) 8261-8269. doi:10.1021/bi00421a041.

[31] M. Chen, M. Li, C. L. Brosseau, J. Lipkowski, AFM Studies of the Effect of Temperature and Electric Field on the Structure of a DMPC-Cholesterol Bilayer Supported on a Au (111) Electrode Surface, Langmuir. 25 (2009) 1028-1037.

[32] C. Li, T. Salditt, Structure of magainin and alamethicin in model membranes studied by xray reflectivity., Biophys. J. 91 (2006) 3285-3300. doi:10.1529/biophysj.106.090118.

[33] H.J. Butt, B. Cappella, M. Kappl, Force measurements with the atomic force microscope: Technique, interpretation and applications, Surf. Sci. Rep. 59 (2005) 1-152. doi:10.1016/j.surfrep.2005.08.003.

[34] H.J. Butt, V. Franz, Rupture of molecular thin films observed in atomic force microscopy. I. Theory, Phys. Rev. E - Stat. Nonlinear, Soft Matter Phys. 66 (2002). doi:10.1103/PhysRevE.66.031601.

[35] S. Loi, G. Sun, V. Franz, H. J. Butt, Rupture of molecular thin films observed in atomic force microscopy. II. Experiment. Phys. Rev. E. 66 (2002), 031602..

[36] Y.F. Dufrene, J.W. Schneider, W.R. Barger, U. Lee, Y.F. Dufrêne, T. Boland, J.W. Schneider, W.R. Barger, G.U. Lee, Characterization of the physical properties of model biomembranes at the nanometer scale with the atomic force microscope., Faraday Discuss. (1998) 57-79. doi:10.1039/a807637e.

[37] J.M. Wenda, J. Juhaniewicz, D. Tymecka, D. Konarzewska, S. Sęk, Modulation of Activity of Ultrashort Lipopeptides toward Negatively Charged Model Lipid Films, Langmuir. 33 (2017) 4619-4627. doi:10.1021/acs.langmuir.6b04674.

[38] B. Gumí-Audenis, F. Sanz, M.I. Giannotti, Impact of galactosylceramides on the nanomechanical properties of lipid bilayer models: an AFM-force spectroscopy study, Soft Matter. 11 (2015) 5447-5454. doi:10.1039/C5SM01252J.

[39] S. Garcia-Manyes, F. Sanz, Nanomechanics of lipid bilayers by force spectroscopy with AFM: A perspective, Biochim. Biophys. Acta - Biomembr. 1798 (2010) 741-749. doi:10.1016/j.bbamem.2009.12.019.

[40] S. Garcia-Manyes, L. Redondo-Morata, G. Oncins, F. Sanz, Nanomechanics of lipid bilayers: Heads or tails?, J. Am. Chem. Soc. 132 (2010) 12874-12886. doi:10.1021/ja1002185.

[41] G. Valincius, M. Mickevicius, Tethered Phospholipid Bilayer Membranes. An Interpretation of the Electrochemical Impedance Response, 1st ed., Elsevier Inc., 2015. doi:10.1016/bs.adplan.2015.01.003.

[42] G. Valincius, T. Meškauskas, F. Ivanauskas, Electrochemical impedance spectroscopy of tethered bilayer membranes, Langmuir. 28 (2012) 977-990. doi:10.1021/la204054g.

[43] E. Madrid, S.L. Horswell, Effect of electric field on structure and dynamics of bilayers formed from anionic phospholipids, Electrochim. Acta. 146 (2014) 850-860. doi:10.1016/j.electacta.2014.01.035.

[44] S. Sek, T. Laredo, J.R. Dutcher, J. Lipkowski, Molecular resolution imaging of an antibiotic peptide in a lipid matrix, J. Am. Chem. Soc. 131 (2009) 6439-6444. doi:10.1021/ja808180m.

[45] N. Garcia-Araez, C.L. Brosseau, P. Rodriguez, J. Lipkowski, Layer-by-layer PMIRRAS characterization of DMPC bilayers deposited on a $\mathrm{Au}(111)$ electrode surface, Langmuir. 22 (2006) 10365-10371. doi:10.1021/la061217v. 
[46] D. Constantin, G. Brotons, A. Jarre, C. Li, T. Salditt, Interaction of alamethicin pores in DMPC bilayers., Biophys. J. 92 (2007) 3978-3987. doi:10.1529/biophysj.106.101204.

[47] J. Breed, I.D. Kerr, R. Sankararamakrishnan, M.S.P. Sansom, Packing interactions of aibcontaining helices: Molecular modeling of parallel dimers of simple hydrophobic helices and of alamethicin, Biopolymers. 35 (1995) 639-655. doi:10.1002/bip.360350610.

[48] D. Peter Tieleman, H.J.C. Berendsen, M.S.P. Sansom, An Alamethicin Channel in a Lipid Bilayer: Molecular Dynamics Simulations, Biophys. J. 76 (1999) 1757-1769. doi:10.1016/S0006-3495(99)77337-6.

[49] D.P. Tieleman, B. Hess, M.S.P. Sansom, Analysis and evaluation of channel models: simulations of alamethicin., Biophys. J. 83 (2002) 2393-2407. doi:10.1016/S00063495(02)75253-3. 
